\title{
Radiative and Electroweak $B$ Decays at the LHC
}

\author{
S. de Capua ${ }^{\mathrm{a}}$ (on behalf of the ATLAS, CMS and LHCb Collaborations) \\ ${ }^{a}$ Università di Roma Tor Vergata and INFN Roma Tor Vergata, \\ Via della Ricerca Scientifica 1, I-00133 Rome, Italy
}

Rare loop-induced decays are sensitive to new physics in many Standard Model extensions. In this paper we discuss the potential of the LHC experiments to study the radiative penguin decays $b \rightarrow s \gamma$ and the electroweak penguin decays $b \rightarrow \ell \ell s$. The experimental strategies are presented.

\section{INTRODUCTION}

Although the Standard Model (SM) is successful in explaining almost all experimental results of elementary particle physics, it is possible that physics beyond the SM exists just above the presently available energy scale. In this paper we will focus on the flavor-changing neutral currents (FCNCs) $b \rightarrow s$ processes, which are forbidden in the Standard Model at the tree level and can proceed only via loop diagrams. If additional diagrams with non-SM particles contribute to these processes, the decay rate and other properties are modified. Such contributions may change the Wilson coefficients that parametrize the strength of the short distance interactions [1]. Hence, studies of the rare radiative penguin decays $b \rightarrow s \gamma$ and the electroweak penguin decay $b \rightarrow \ell \ell s$ could allow an extraction of information sensitive to new physics in many SM extensions.

The radiative penguin $b \rightarrow s \gamma$ decay is a prime example of remarkable interest. Its total branching fraction is very sensitive to physics beyond the SM as it may be affected by the presence of charged Higgs or SUSY particles in the loop. But the present theoretical prediction for the branching fraction of $(3.55 \pm 0.26) \times 10^{-4}[2,3]$, and the world average experimental value $(3.7 \pm 0.3) \times$ $10^{-4}$ [4] agree well. Nevertheless, the helicity of the photon emitted in the $b \rightarrow s \gamma$ decay is still an untested prediction of the SM in the $B$ meson sector [5]. The photon in radiative $\bar{B}^{0}$ and $\bar{B}_{s}^{0}$ decays is expected to be predominantly left-handed. Thus, mixing-induced CP asymmetries in $b \rightarrow s \gamma$ are suppressed (by the ratio of the quark masses $m_{s} / m_{b}$ ) and are very small. In many extensions of the SM the amplitude of right-handed photons grows proportional to the virtual heavy fermion mass, which could lead to angular asymmetries of the decay products as large as $50 \%$. Given the experimental difficulty of a direct measurement of the photon helicity in $B$ meson decays, several indirect methods for its determination have been proposed [6-10].

Measurements of the forward- backward asymmetry $A_{F B}$, defined for the transition $b \rightarrow \ell \ell s$ through the angle $\theta_{F B}$ between the $\ell^{+}$and the $b$ hadron flight directions in the di-lepton rest frame, and differential decay rate as functions of the squared invariant mass of the dilepton system $q^{2}$ and $\theta_{F B}$, constrains the relative signs and magnitudes of some of the Wilson coefficients. In particular, the shape of the asymmetry $A_{F B}$ as a function of lepton-lepton effective mass $m_{\ell \ell}^{2}$ and especially the position of the zero crossing (i.e. the $m_{\ell \ell}^{2}$ value corresponding to $\left.A_{F B}=0\right)$ are almost unaffected by hadronic form factor uncertainties, thus providing a good basis for searching for deviations from SM predictions [11]. Finally, the ratio of $b \rightarrow \mu \mu s$ and $b \rightarrow e e s$ decays in any exclusive mode is also a clean probe of the SM. Lepton-universality predicts this ratio to be unity with theoretical errors below $1 \%$ [12].

The LHC will be a copious source of $B$ mesons, due to the expected high $b \bar{b}$ cross section of $\sim 500 \mu \mathrm{b}$. This potential will be exploited by the ATLAS, CMS and LHCb experiments. The ATLAS and CMS detectors are multi-purpose cen- 
tral spectrometers and they have been designed primarly for new physics searches at high luminosity $[13,14]$. Nevertheless they have the capabilities to realise a rich and competitive programme dedicated to $B$ physics [15] for decays involving muons during the initial running at lower luminosity and especially the ATLAS collaboration has recently reported results of various studies dedicated to $B$ physics. We assume for the following that this programme covers three years of running at luminosity $\mathcal{L}=10^{33} \mathrm{~cm}^{-2} \mathrm{~s}^{-1}$, i.e. $30 \mathrm{fb}^{-1}$ integrated luminosity.

$\mathrm{LHCb}$ is a forward spectrometer optimised for $B$ physics. Its main features are a precise vertex detector, two RICH detectors and a versatile trigger with a $2 \mathrm{kHz}$ output rate dominated by $b \bar{b}$ events. $\mathrm{LHCb}$ will operate at a lower luminosity of $\mathcal{L}=2 \cdot 10^{32} \mathrm{~cm}^{-2} \mathrm{~s}^{-1}$, corresponding to $2 \mathrm{fb}^{-1}$ per year.

\section{RADIATIVE RARE DECAYS AT THE LHC}

In the LHCb experiment, radiative $b \rightarrow s \gamma$ decays can be reconstructed in the modes $B^{0} \rightarrow$ $K^{0 *} \gamma$ and $B_{s}^{0} \rightarrow \phi \gamma[16]$. (Throughout this paper, unless specified otherwise, charge-conjugated modes are implied). The reconstruction procedures for $B^{0} \rightarrow K^{0 *} \gamma$ (followed by $K^{0 *} \rightarrow$ $K^{+} \pi^{-}$) and $B_{s}^{0} \rightarrow \phi \gamma\left(\right.$ followed by $\phi \rightarrow K^{+} K^{-}$) decays are similar and the off-line selection criteria will be described for both channels in parallel. Charged tracks have to be consistent with the requested particle identification and inconsistent with originating from the reconstructed primary vertex. Selected $\mathrm{K}^{* 0}(\phi)$ candidates are combined with photon candidates of transverse energy greater than $2.8 \mathrm{GeV} / c^{2}$, in order to remove low energy $\gamma$ and $\pi^{0}$ s. The reconstructed $B$ candidate is required to be compatible with coming from a primary vertex. This requirement is one of the most powerful cuts against combinatorial background. Finally, to suppress the correlated background from the decays $B^{0} \rightarrow K^{* 0} \pi^{0}$ and $B_{s}^{0} \rightarrow \phi \pi^{0}$, a cut on the $K^{* 0}$ and $\phi$ decay (helicity) angle with respect to the $B$ direction is applied. The mass distributions of the selected and triggered $B$ candidates are shown in

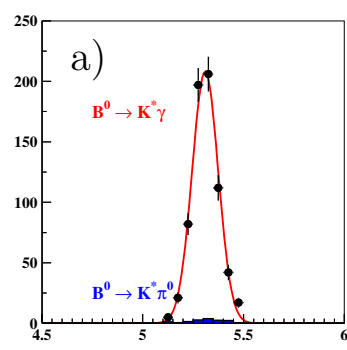

$$
\mathrm{m}_{\mathrm{K}^{* 0} \gamma}\left[\mathrm{GeV} / c^{2}\right]
$$

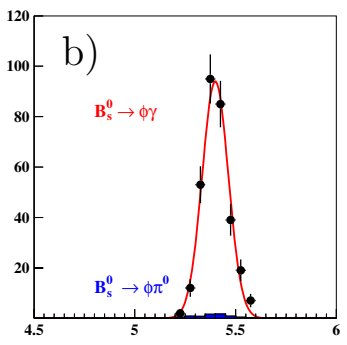

$\mathrm{m}_{\phi \gamma}\left[\mathrm{GeV} / c^{2}\right]$

Figure 1. a) $B^{0} \rightarrow K^{0 *} \gamma$ and b) $B_{s}^{0} \rightarrow \phi \gamma$ reconstructed mass distributions for signal events in $\mathrm{LHCb}$ after trigger and selection cuts. The specific backgrounds from $B^{0} \rightarrow K^{* 0} \pi^{0}$ and $B_{s}^{0} \rightarrow$ $\phi \pi^{0}$ decays are shown with correct relative normalisation. The dominant combinatorial background is not shown.

Fig. 1; the mass resolutions are expected to be $(64 \pm 2) \mathrm{MeV} / c^{2}$ and $(65 \pm 4) \mathrm{MeV} / c^{2}$ respectively.

The expected annual $\left(2 \mathrm{fb}^{-1}\right)$ yields and ratios of background over signal $(B / S)$ are given in Table 1. The background is estimated from a fully simulated $b \bar{b}$ Monte Carlo (MC) sample. The limits are given at the $90 \%$ confidence level. These yields will also allow CP asymmetry measurements in $B^{0} \rightarrow K^{0 *} \gamma$ decays with a statistical accurancy at the per-cent level.

In the ATLAS experiment, the reconstruction procedure consists of requiring a muon with transverse momentum, $p_{t}$, higher than $6 \mathrm{GeV} / c$, together with a Region Of Interest (ROI) in the electromagnetic calorimeter with a transverse energy larger than $5 \mathrm{GeV} / c^{2}$. The achieved $B$ mass resolution is greater than $100 \mathrm{MeV} / c^{2}$, but it can be improved with an optimization of the offline cuts. The expected event yields [17] after 2 years $\left(20 \mathrm{fb}^{-1}\right)$ are reported in Table 2 . These results are limited by the background statistics and better signal to background ratios can be reached in future analyses. 
Table 1

Annual yields and background-to-signal ratios for selective radiative $B$ meson and baryon and electroweak decays at $\mathrm{LHCb}$.

\begin{tabular}{lrc}
\hline Mode & Yield & $B / S$ \\
\hline \hline$B$ meson decays & & \\
\hline$B^{0} \rightarrow K^{0 *} \gamma$ & 35000 & $<0.7$ \\
$B_{s}^{0} \rightarrow \phi \gamma$ & 9000 & $<2.4$ \\
\hline \hline$B$ baryon decays & & \\
\hline$\Lambda_{b} \rightarrow \Lambda \gamma$ & 750 & $<42$ \\
\hline$\Lambda_{b} \rightarrow \Lambda(1520) \gamma$ & 4200 & $<10$ \\
$\Lambda_{b} \rightarrow \Lambda(1670) \gamma$ & 2500 & $<18$ \\
$\Lambda_{b} \rightarrow \Lambda(1690) \gamma$ & 2200 & $<18$ \\
\hline \hline Electroweak decays & & \\
\hline$B^{0} \rightarrow \mu \mu K_{s}^{0}$ & 17500 & $\sim 3$ \\
$B^{0} \rightarrow e e K_{s}^{0}$ & 8740 & $\sim 5$ \\
\hline
\end{tabular}

\section{3. $\Lambda_{b} \rightarrow \Lambda \gamma$ POLARIZATION MEASURE- MENTS AT LHCb}

Radiative decays of polarized $\Lambda_{b}$ baryons decaying to $\Lambda_{b} \rightarrow \Lambda \gamma$ represent an attractive possibility to measure the helicity of the photon emitted in the $b \rightarrow s$ quark transition [18]. From the experimental point of view, the observable of interest is the photon asymmetry

$$
\alpha_{\gamma}=\frac{P\left(\gamma_{L}\right)-P\left(\gamma_{R}\right)}{P\left(\gamma_{L}\right)+P\left(\gamma_{R}\right)}
$$

where $P\left(\gamma_{L(R)}\right)$ represents the probability of producing a left(right)-handed photon. This asymmetry can be measured through the angular distribution of the photon in the $\Lambda_{b}$ rest frame,

$$
\frac{d \Gamma}{d \cos \theta_{\gamma}} \propto 1-\alpha_{\gamma} P_{\Lambda_{b}} \cos \theta_{\gamma}
$$

where $P_{\Lambda_{b}}$ is the $\Lambda_{b}$ polarization, or even through the angular distribution of the proton coming from the $\Lambda \rightarrow p \pi^{-}$decay in the $\Lambda$ rest frame:

$$
\frac{d \Gamma}{d \cos \theta_{p}} \propto 1-\alpha_{\gamma} \alpha_{p} \cos \theta_{p}
$$

where $\alpha_{p}$ is the proton asymmetry, $\theta_{p}$ is the angle between the proton and the $\Lambda_{b}$ flight directions.
Table 2

Annual yields and background-to-signal ratios for selective radiative $B$ meson and electroweak decays at ATLAS.

\begin{tabular}{lrr}
\hline Mode & Yield & $B / S$ \\
\hline \hline$B$ meson decays & & \\
\hline$B^{0} \rightarrow K^{0 *} \gamma$ & 9400 & $<100$ \\
$B_{s}^{0} \rightarrow \phi \gamma$ & 3200 & $<25$ \\
\hline \hline Electroweak decays & & \\
\hline$B^{0} \rightarrow \mu \mu K^{0 *}$ & 2500 & $<20$ \\
$B^{+} \rightarrow \mu \mu K^{+}$ & 1500 & $<6$ \\
$B_{s}^{0} \rightarrow \mu \mu \phi$ & 900 & $<11$ \\
$\Lambda_{b} \rightarrow \mu \mu \Lambda$ & 800 & $<5$ \\
\hline
\end{tabular}

The study of this channel is challenging at the LHC because of the long-length decay of the $\Lambda$ baryon, which typically traverses a large fraction of the tracking system before decaying. A possible way around this problem is given by considering decays to heavier $\Lambda$ resonances; with their decay into $\pi K^{-}$, they can trace back the decay of the $\Lambda_{b}$ [19].

The event selection is similar to the one presented above for $B$ mesons. In a preliminary study $\mathrm{LHCb}$ expects the yields and $B / S$ ratios for $2 \mathrm{fb}^{-1}$ as given in Table 1 . As shown in the table, the heavier $\Lambda$ modes have a higher statistical power, but since the proton polarization is expected to be a flat distribution (i.e. the proton asymmetry is uniform $\alpha_{p}=0$ due to parity conservation), the intrinsic sensitivity is lower.

We can extract $\alpha_{\gamma}$ from Eq. 2, measuring the $\Lambda_{b}$ polarization from $\Lambda_{b} \rightarrow \Lambda \mathrm{J} / \psi$ with a precision of $1 \%$ [20]. With these yields $\mathrm{LHCb}$ will be able to measure the right-handed polarisation component to an accuracy of $5 \%$ with $10 \mathrm{fb}^{-1}$ (five years of data taking).

\section{FORWARD-BACKWARD ASYMME- TRY}

Because of its very clean experimental signature, the decay $b \rightarrow \mu \mu s$ can be accessed by ATLAS, CMS and LHCb in the exclusive decays $\Lambda_{b} \rightarrow \mu \mu \Lambda, B^{+} \rightarrow \mu \mu K^{+}, B^{0} \rightarrow \mu \mu K^{0 *}$ and 

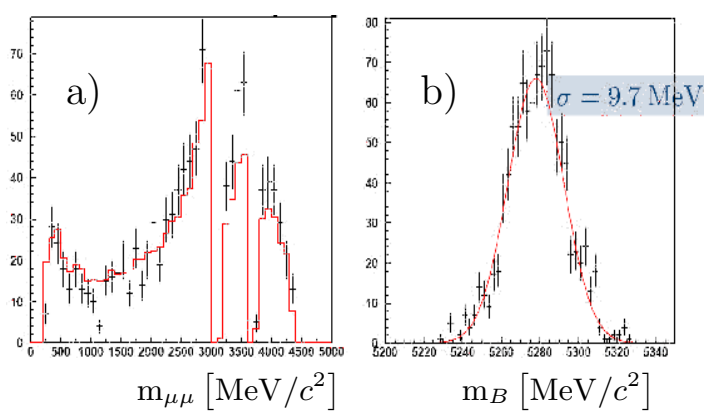

Figure 2. a) Comparison of the $\mu \mu$ invariant mass distribution for selected events (points) and for the true MC events (continuous histogram). b) The reconstructed $B$ mass in $B^{0} \rightarrow \mu \mu K^{0 *}$ events at $\mathrm{LHCb}$.

$B_{s}^{0} \rightarrow \mu \mu \phi$. The selections combine two tracks positively identified as opposite-charged muons with the relevant hadronic final state. Similar selection criteria as in Section 2 are applied. Very strict requirements on the vertex quality are applied to reduce the backgrounds from cascade semileptonic $b \rightarrow \mu \nu c, c \rightarrow \mu \nu s$ decays and from two semileptonic $b \rightarrow \mu \nu c$ decays. These processes need to be well under control because they can induce an $A_{F B}$ bias. The background from $c \bar{c}$ resonances is removed by vetoing the $J / \psi$ and $\psi(2 S)$ mass windows in the $(\mu \mu)$ effective mass distribution.

Regarding the decay $B^{0} \rightarrow \mu \mu K^{0 *}$, LHCb expects a $15 \mathrm{MeV} / c^{2}$ resolution for the $B$ mass and $10 \mathrm{MeV} / c^{2}$ for the $\mu \mu$ mass (see Fig. 2). The resolution for $\theta_{F B}$ is $4 \mathrm{mrad}$. The distributions for the di-muon invariant mass and the angle $\theta_{F B}$ are not distorted by acceptance or selection cuts [21]. The expected yield for one nominal year of running at $\mathrm{LHCb}\left(2 \mathrm{fb}^{-1}\right)$ for the channel $B^{0} \rightarrow \mu \mu K^{0 *}$ (assuming the SM branching ratio of $12 \times 10^{-7}$ ) is about 7300 reconstructed events with a background to signal ratio $B / S \simeq 1$. The level of all the possible background categories and how to deal with them is at present under study.

Using the results obtained from the full simulation of the $B^{0} \rightarrow \mu \mu K^{0 *}$ channel,

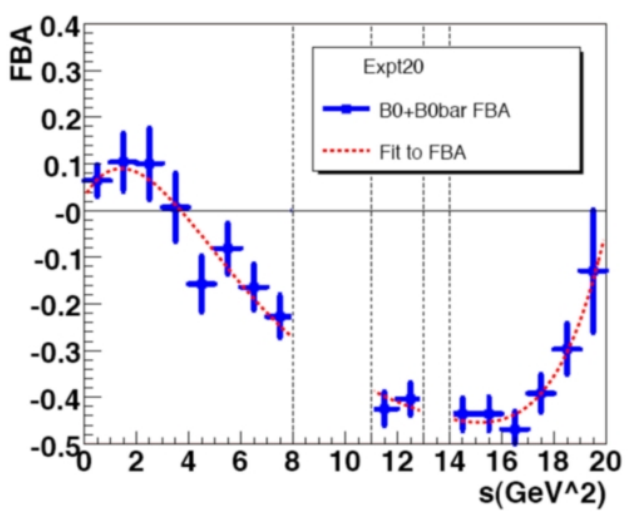

Figure 3. Typical $A_{F B}$ versus di-lepton mass squared for the decay $B^{0} \rightarrow \mu \mu K^{0 *}$ with $2 \mathrm{fb}^{-1}$ of integrated luminosity at $\mathrm{LHCb}$.

$\mathrm{LHCb}$ has estimated the sensitivity to the forward - backward asymmetry in a "toy" MC study. Typical behavior of $A_{F B}$ versus $\mathrm{m}_{\mu \mu}^{2}$, after one year of running at the nominal luminosity $\left(2 \mathrm{fb}^{-1}\right)$ is demonstrated in Fig. 3. $\mathrm{LHCb}$ expects to extract the $C_{9} / C_{7}$ Wilson-coefficients ratio from the crossing point with the $A_{F B}=0$ axis to a precision of $13 \%$ in $10 \mathrm{fb}^{-1}$ of data (five years of running).

A study of the $B^{0} \rightarrow \mu \mu K^{0 *}$ channel in ATLAS has also been carried out, and the expectation is to reconstruct about 1400 events in $20 \mathrm{fb}^{-1}$ (two years of running), with a background to signal ratio of about 10 [22].

Also in ATLAS, a study of the channel $\Lambda_{b} \rightarrow$ $\mu \mu \Lambda$ has been recently performed [23], a channel which provides a similar phenomenology as the $B^{0} \rightarrow \mu \mu K^{0 *}$ decay. The $\Lambda$ is selected requiring that the radial coordinate of the $\Lambda_{b} \rightarrow \mu \mu \Lambda$ vertex is in the range $1-45 \mathrm{~cm}$ and that the $\Lambda_{b}$ proper time is larger than 0.5 ps. ATLAS expects a $75 \mathrm{MeV} / c^{2}$ resolution for the $\Lambda_{b}$ mass, and should also be able to disentangle the SM expectation from new physics with positive Wilson coefficient $C_{7}$ in $30 \mathrm{fb}^{-1}$ of data. A summary of the ATLAS potential in the electroweak sector is listed in Table 2 for three years of running at 
$\mathcal{L}=10^{33} \mathrm{~cm}^{-2} \mathrm{~s}^{-1}\left(30 \mathrm{fb}^{-1}\right)$.

\section{5. $R_{\mathrm{K}}$ MEASUREMENTS AT LHCb}

In the SM, the ratio of $b \rightarrow \mu \mu s$ and $b \rightarrow$ ees decays is expected to be very close to unity, namely $R_{\mathrm{K}}=1.000 \pm 0.001$ and $R_{\mathrm{K}^{0 *}}=0.991 \pm 0.002$ [12]. Deviations of order of $10 \%$ can occur with neutral Higgs boson exchange in models that distinguish between lepton flavours (for instance, minimal SUSY model at large $\tan \beta$ ).

In LHCb, a study of the channel $B^{+} \rightarrow$ $\mu \mu K^{+}$as well as $B^{+} \rightarrow e e K^{+}$has been recently performed [24]. The reconstruction of these two decay modes allows an extraction of the ratio $R_{\mathrm{K}}$ of the two branching fractions, integrated over a given di-lepton mass range. $B^{+} \rightarrow \mu \mu K^{+}$and $B^{+} \rightarrow e e K^{+}$decays are reconstructed with the same requirements and with the same procedure explained above, except that a proper bremsstrahlung correction is essential in the $B^{+} \rightarrow e e K^{+}$channel (see [24] for details).

The di-lepton mass range is chosen to be $1<$ $m_{\ell \ell}^{2}<6 \mathrm{GeV}^{2} / \mathrm{c}^{4}$ in order to avoid $c \bar{c}$ resonances. The event yields are extracted from a twodimensional fit to the $\ell \ell K$ and $\ell \ell$ masses in order to take into account the backgrounds from $b \rightarrow$ $\mathrm{J} / \psi s$ and $B^{0} \rightarrow \ell \ell K^{0 *}$.

The expected $B$ candidate mass distributions are shown in Fig. 4 for five years $\left(10 \mathrm{fb}^{-1}\right)$ of $\mathrm{LHCb}$ data taking. The yields are given in Table 1 . Assuming only statistical errors, an error on $R_{K}$ of $4 \%$ is achieved after five years of running.

\section{CONCLUSIONS}

The LHC experiments have promising potential for the study of rare loop-induced decays sensitive to new physics in many Standard Model extensions. In particular, the capabilities to measure the helicity of the photon emitted in the $b \rightarrow s \gamma$ decay, the forward-backward asymmetry $A_{F B}$ for the transition $b \rightarrow \ell \ell s$ and the ratio of $b \rightarrow \mu \mu s$ and $b \rightarrow e e s$ decays in a number of exclusive modes has been studied. The experimental strategies, and the expected annual signal yields and background ratios have been presented.
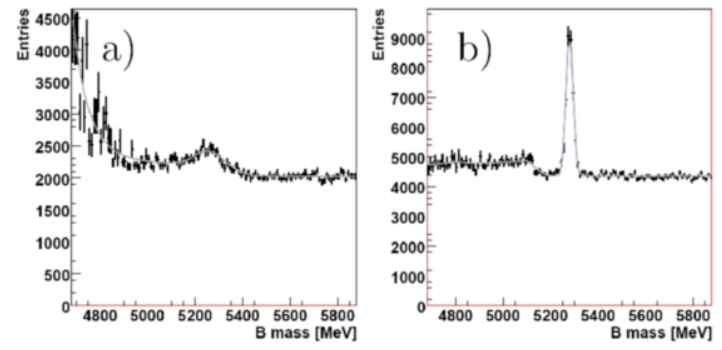

Figure 4. Expected $B$ candidate mass distributions in a) $B^{+} \rightarrow e e K^{+}$and b) $B^{+} \rightarrow \mu \mu K^{+}$ modes for $10 \mathrm{fb}^{-1}$ of data at LHCb.

\section{REFERENCES}

1. G. Buchalla et al., Rev. Mod. Phys. 68 (1996) 1125.

2. T. Hurth et al., Nucl. Phys. B 704 (2003) 56.

3. P. Gambino and M. Misiak, Nucl. Phys. B 611 (2001) 338.

4. W.-M. Yao et al. (Particle Data Group), Journal of Physics G 33 (2006) 1.

5. D. Atwood et al., Phys. Rev. Lett. 79 (1997) 185.

6. D. Melikhov et al., Phys. Lett. B 442 (1998) 381.

7. Y. Grossman and D. Pirjol, JHEP 0006 (2000) 029.

8. M. Gronau and D. Pirjol, Phys. Rev. D 66 (2002) 054008.

9. M. Gronau et al., Phys. Rev. Lett. 66 (2002) 051802.

10. G. Hiller and A. Kagan, Phys. Rev. D 65 (2002) 074038.

11. A. Ali, ICHEP 2002, hep-ph/0210183.

12. G. Hiller and F. Krüger, Phys. Rev. D 69 (2004) 074020.

13. The ATLAS Technical Proposal, CERN/LHCC/94-43, LHCC/P2 (1994).

14. The CMS Technical Proposal, CERN/LHCC/94-38, LHCC/P1 (1994).

15. P. Ball et al., hep-ph/0003238.

16. G. Pakhlova and I. Belyaev, LHCb-2003-090. 
17. S. Viret, ATLAS phys-pub-2005-006.

18. T. Mannel and S. Recksiegel, Nucl. Part. Phys 24 (1998) 979.

19. F. Legger, T. Schietinger, hep-ph/0605245.

20. J. Hřivnáč et al. J. Phys. G 21 (1995) 629.

21. J.H. Lopes, LHCb-2003-104.

22. S. Viret, ATLAS phys-conf-2005-005.

23. N. Nikitin et al., Nucl. Phys. B (Proc. Suppl.) 156 (2006) 119.

24. P. Koppenburg, talk given at the 7th International Conference on Hyperons, Charm And Beauty Hadrons, Lancaster (2006). To be published in Nucl. Phys. B (Proc. Suppl.) 